David S. Y. Ong

Cristian Spitoni

Peter M. C. Klein Klouwenberg

Frans M. Verduyn Lunel

Jos F. Frencken

Marcus J. Schultz

Tom van der Poll

Jozef Kesecioglu

Marc J. M. Bonten

Olaf L. Cremer

\title{
Cytomegalovirus reactivation and mortality in patients with acute respiratory distress syndrome
}

Received: 14 July 2015

Accepted: 12 September 2015

Published online: 28 September 2015

(C) The Author(s) 2015. This article is published with open access at Springerlink.com

Take-home message: CMV-seropositive critically ill patients with ARDS who remain mechanically ventilated for more than 4 days have a $27 \%$ cumulative risk of developing CMV reactivation while in the ICU. Despite extensive statistical correction to account for time-varying aspects of exposure, competing risks, and the presence of confounding by differences in the evolution of disease severity prior to viremia onset, CMV reactivation remains independently associated with increased case fatality. The absolute mortality due to these reactivations is estimated at $4.4 \%$ (95\% CI 1.1-7.9) by day 30 .

On behalf of the MARS consortium. The members of the MARS consortium are given in the "Appendix".

Electronic supplementary material The online version of this article (doi:10.1007/s00134-015-4071-z) contains supplementary material, which is available to authorized users.
D. S. Y. Ong (®) •

P. M. C. Klein Klouwenberg ·

F. M. Verduyn Lunel - M. J. M. Bonten

Department of Medical Microbiology,

University Medical Center Utrecht, Utrecht,

The Netherlands

e-mail: davidsyong@gmail.com

Tel.: +3188756 1124
D. S. Y. Ong - P. M. C. Klein Klouwenberg J. F. Frencken - J. Kesecioglu - O. L. Cremer Department of Intensive Care Medicine, University Medical Center Utrecht, Utrecht, The Netherlands

\section{S. Y. Ong - C. Spitoni .}

P. M. C. Klein Klouwenberg .

J. F. Frencken - M. J. M. Bonten

Julius Center for Health Sciences and

Primary Care, University Medical Center

Utrecht, Utrecht, The Netherlands

\section{Spitoni}

Department of Mathematics, Utrecht

University, Utrecht, The Netherlands

\section{J. Schultz}

Department of Intensive Care, Academic Medical Center, University of Amsterdam, Amsterdam, The Netherlands

T. van der Poll

Center of Experimental and Molecular Medicine and Division of Infectious Diseases, Academic Medical Center, University of Amsterdam, Amsterdam, The Netherlands

\section{T. van der Poll}

Center for Infection and Immunity Amsterdam, Academic Medical Center, University of Amsterdam, Amsterdam, The Netherlands

Abstract Purpose: Cytomegalovirus (CMV) reactivation occurs frequently in patients with the acute respiratory distress syndrome (ARDS) and has been associated with increased mortality. However, it remains unknown whether this association represents an independent risk for poor outcome. We aimed to estimate the attributable effect of CMV reactivation on mortality in immunocompetent ARDS patients. Methods: We prospectively studied immunocompetent ARDS patients who tested seropositive for CMV and remained mechanically ventilated beyond day 4 in two tertiary intensive care units in the Netherlands from 2011 to 2013. CMV loads were determined in plasma weekly. Competing risks Cox regression was used with CMV reactivation status as a time-dependent exposure variable. Subsequently, in sensitivity analyses we adjusted for the evolution of disease severity until onset of reactivation using marginal structural modeling. Results: Of 399 ARDS patients, 271 (68\%) were CMV seropositive and reactivation occurred in $74(27 \%)$ of them. After adjustment for confounding and competing risks, CMV reactivation was associated with overall increased ICU mortality (adjusted subdistribution hazard ratio (SHR) 2.74, $95 \% \mathrm{CI}$ 1.51-4.97), which resulted from the joint action of trends toward an increased mortality rate (direct effect; cause specific hazard ratio (HR) 1.58 , $95 \%$ CI 0.86-2.90) and a reduced successful weaning rate (indirect effect; cause specific HR $0.83,95 \%$ CI 0.58-1.18). These associations remained in sensitivity analyses. The 
population-attributable fraction of ICU mortality was $23 \%$ (95\% CI $6-41$ ) by day 30 (risk difference 4.4 , $95 \%$ CI 1.1-7.9). Conclu-

sion: CMV reactivation is

independently associated with increased case fatality in immunocompetent ARDS patients who are CMV seropositive.
Keywords Cytomegalovirus . Viremia - Reactivation · ARDS . Mortality

\section{Introduction}

Although the burden of cytomegalovirus (CMV) disease has been well established in immunocompromised patients [1], CMV viremia has also been described in intensive care unit (ICU) patients without known prior immune deficiency. This almost exclusively results from systemic viral reactivation, and incidence rates of up to $40 \%$ have been reported in critically ill CMV seropositive subjects [2-9]. Furthermore, CMV reactivation in critically ill patients has been associated with a prolonged duration of mechanical ventilation $[2,4,9-13]$, an increased length of stay in the ICU $[3,5,9,10,13]$, and excess mortality [2, 4, 7-9]. Nevertheless, it remains uncertain whether these findings imply that CMV reactivation is a truly independent risk factor with respect to these observed poor clinical outcomes because most studies that have assessed these associations did not adequately account for all possible sources of bias. As a consequence, CMV viremia might merely be a marker of illness severity, contributing only little to the overall burden of disease.

To achieve an accurate estimation of the true effect of CMV reactivation on clinical outcome, it is crucial in observational studies to adjust for the time-dependent occurrence of CMV reactivation and the evolution of disease severity prior to its onset. Moreover, the presence of competing events should be taken into account when follow-up time is censored [14]. For instance, when ICU mortality is the outcome, then ICU discharge is a competing risk that prohibits the event of interest from occurring first.

Patients with acute respiratory distress syndrome (ARDS) often have a long and complicated disease course in the ICU, which portends a particular risk for viral reactivations $[15,16]$. Despite the uncertainties regarding the clinical relevance of CMV disease in immunocompetent critically ill patients, it is etiologically plausible that virus reactivation adds to the pulmonary pathology in patients with ARDS. In experimental murine studies, CMV reactivation caused exacerbated and prolonged cytokine and chemokine expression in lung tissues, which eventually led to increased pulmonary fibrosis compared to controls [17]. In a clinical study of open lung biopsies in ARDS patients with prolonged respiratory failure or in whom microbiological cultures remained negative, CMV pneumonia was found in $30 \%$ of cases [18]. Both findings suggest that CMV-related pulmonary pathology may be causally linked to the clinical disease course following ARDS onset, especially in the most severely ill patients who require prolonged mechanical ventilation. If CMV reactivation does contribute to poor clinical outcome in these patients, either prophylaxis or pre-emptive therapy with (val)ganciclovir may be considered.

The aim of this study was to estimate the proportion of deaths that can be attributed to systemic reactivation of CMV in ARDS patients who are latent carriers of the virus. Some results of this study have been previously reported in the form of an abstract [19].

\section{Methods}

Patients and measurements

The present study was conducted within the framework of the Molecular Diagnosis and Risk Stratification of Sepsis (MARS) cohort (clinicaltrials.gov identifier: NCT01905033) for which the institutional review board approved an opt-out method of informed consent (protocol number 10-056C) [20]. We prospectively included consecutive adults who presented with ARDS to the mixed ICUs of two tertiary care hospitals in the Netherlands between January 2011 and December 2013 and required mechanical ventilation beyond day 4 of ICU admission. Since data collection for our study started before publication of the Berlin definition in 2012, ARDS was defined according to the American-European Consensus Conference criteria [21]: that is, the diagnosis required an acute onset of symptoms, the presence of bilateral infiltrates on chest radiography, a pulmonary artery occlusion pressure less than $18 \mathrm{mmHg}$ and/or the absence of left ventricular dysfunction, and $\mathrm{PaO}_{2} / \mathrm{FiO}_{2}$ ratio $(\mathrm{P} / \mathrm{F})$ less than 300 . We excluded patients who had received (val)ganciclovir, (val)acyclovir, cidofovir, or foscarnet in the week before ICU admission and those with known immunodeficiency [16]. Immunodeficiency was defined as a history of solid organ or stem cell transplantation, infection with the human immunodeficiency virus, hematological malignancy, use of immunosuppressive medication (more than $0.1 \mathrm{mg}$ prednisone per kilo for more than 3 months, more than $75 \mathrm{mg}$ prednisone per day for more than 1 week, or equivalent), 
chemotherapy/radiotherapy in the year before ICU admission, and any known humoral or cellular immune deficiency.

Leftover plasma, which was harvested from blood samples obtained daily as part of routine patient care, was stored at $-80{ }^{\circ} \mathrm{C}$ within $4 \mathrm{~h}$ after blood draw. CMV serostatus was determined by an enzyme immunoassay (Enzygnost CMV/IgG, Siemens Healthcare Diagnostic Products, Marburg, Germany). Subsequently, in seropositive patients only, viral loads in plasma were determined by real-time Taqman CMV-DNA polymerase chain reaction [22]. CMV loads were determined on a weekly basis for a maximum of 30 days following study inclusion (i.e., day 5 of ICU admission). For intermediary days, on which quantitative PCR was not performed, we estimated viral loads by log-linear imputation. CMV reactivation was defined as a viral load of at least 100 international units per milliliter (IU/mL), as calibrated according to the CMV World Health Organization (WHO) Standard. Screening for CMV was not part of routine clinical practice in either participating hospital. Neither serology results nor viral loads measured as part of our study were made available to the treating physicians, and none of the included patients therefore received antiviral treatment directed against CMV.
Mortality was the outcome of primary interest in this study and was defined as death on mechanical ventilation before day 35 (i.e., day 30 following study inclusion). Successful weaning, which is a competing event of the primary outcome, was defined as complete liberation from mechanical ventilatory support on two or more consecutive days before day 35 . We considered distal end points more likely to be amenable by pre-existing comorbidities, as well as specific end-of-life practices, bed availability, and other local factors. Nonetheless, in a subsequent sensitivity analysis, we used discharge and death in ICU as alternative end points.

\section{Data analysis}

For our primary analyses we used Cox proportional hazards modeling, in which mortality and successful weaning were considered as competing events and CMV reactivation status was fitted as a time-dependent variable. Possible confounders that were screened included all patient characteristics and therapeutic interventions listed in Table 1, and some markers of disease severity: Acute Physiology and Chronic Health Evaluation

Table 1 Characteristics of ARDS patients by CMV reactivation status

\begin{tabular}{|c|c|c|c|}
\hline & Reactivation $(n=74)$ & Non-reactivation $(n=197)$ & $p$ value \\
\hline \multicolumn{4}{|l|}{ Patient characteristics } \\
\hline Age (years) & $64(56-74)$ & $64(54-72)$ & 0.50 \\
\hline Male gender & $44(59)$ & $120(61)$ & 0.83 \\
\hline Non-European descent & $13(18)$ & $27(14)$ & 0.42 \\
\hline Prior ICU admission during hospital stay & $16(22)$ & $24(12)$ & 0.05 \\
\hline Surgical reason for admission & $22(30)$ & $73(37)$ & 0.26 \\
\hline COPD & $13(18)$ & $30(15)$ & 0.64 \\
\hline Congestive heart failure & $2(3)$ & $9(5)$ & 0.49 \\
\hline Diabetes mellitus & $14(19)$ & $26(13)$ & 0.24 \\
\hline Cancer & $9(12)$ & $28(14)$ & 0.66 \\
\hline Renal insufficiency & $12(16)$ & $11(6)$ & $<0.01$ \\
\hline \multicolumn{4}{|l|}{ Markers of disease severity } \\
\hline APACHE IV score & $91(71-113)$ & $76(62-99)$ & $<0.01$ \\
\hline Septic shock & $42(57)$ & $81(41)$ & 0.02 \\
\hline Plasma lactate & $3.6(1.9-6.5)$ & $3.5(1.9-5.8)$ & 0.47 \\
\hline C-reactive protein & $244(129-310)$ & $241(151-309)$ & 0.99 \\
\hline$\geq 3$ quadrant consolidation & $49(66)$ & $121(61)$ & 0.47 \\
\hline Tidal volume (mL/kg body weight) & $6.5(5.5-7.6)$ & $6.5(5.6-7.8)$ & 0.72 \\
\hline PEEP setting $\left(\mathrm{cmH}_{2} \mathrm{O}\right)$ & $12(10-15)$ & $10(8-15)$ & 0.16 \\
\hline $\mathrm{P} / \mathrm{F}$ & $107(77-150)$ & $106(82-149)$ & 0.41 \\
\hline Pulmonary compliance $\left(\mathrm{mL} / \mathrm{cmH}_{2} \mathrm{O}\right)$ & $35(24-47)$ & $39(27-49)$ & 0.15 \\
\hline \multicolumn{4}{|l|}{ Therapeutic interventions } \\
\hline High dose corticosteroid therapy & $48(65)$ & $101(51)$ & 0.05 \\
\hline Transfusion of blood products & $15(20)$ & $18(9)$ & 0.18 \\
\hline
\end{tabular}

Markers of disease severity represent the worst values observed during the first 4 days in ICU, except for tidal volume, which represents the mean value. Therapeutic interventions relate to the first 4 days in ICU only. High dose corticosteroid was defined as a daily dose of $\geq 250 \mathrm{mg}$ hydrocortisone or equivalent

Data are presented as medians (interquartile range) or absolute

APACHE Acute Physiology and Chronic Health Evaluation, ARDS acute respiratory distress syndrome, COPD chronic obstructive pulmonary disease, $I C U$ intensive care unit, $P E E P$ positive end expiratory pressure, $P / F$ partial pressure of oxygen in arterial blood to fraction of inspired oxygen ratio 
(APACHE) IV score, presence of septic shock, partial pressure of oxygen in arterial blood to fraction of inspired oxygen ratio, and positive end expiratory pressure (PEEP) setting. To account for possible confounding, we included baseline covariables that showed differences between the reactivated and non-reactivated groups at a $p$ value of less than 0.30 , and changed the crude effect estimates for either mortality or weaning by more than $10 \%$. We included only the strongest (possible) confounders by using these two criteria combined in order to avoid statistical overfitting (i.e., incorporating too many variables given the limited number of events).

The two possible outcomes are interrelated as increased mortality may negatively impact the duration of mechanical ventilation. A competing risks analysis accommodates for this by providing two measures of association. First, the cause-specific hazard ratio (CSHR) estimates the direct effects of CMV reactivation on each outcome of interest (i.e., mortality on the ventilator and successful weaning). Second, the subdistribution hazard ratio (SHR) estimates the risk of dying from reactivation at a given time-point, while accounting for the competing risk of successful weaning. To obtain direct estimates of cumulative risks in terms of the SHR we used the Fine and Gray model [23]. Finally, to estimate the populationattributable fraction of mortality due to CMV reactivation, we used a multi-state model (Fig. S1), which accounts for the time of reactivation [24]. Confidence intervals were calculated by bootstrap resampling [25, 26].

Despite these efforts to accurately assess the effect of CMV reactivation on clinical outcomes, residual confounding may still remain, because markers of illness at baseline (which we included in all multivariable analyses) may no longer be representative of the disease state at the time of reactivation onset. Thus, we performed a sensitivity analysis using marginal structural modeling to adjust for the evolution of disease severity prior to the onset of CMV reactivation (see also Supplementary Material) [27, 28]. Such analysis first involves estimation of the daily probabilities of CMV reactivation using a multivariable logistic regression model that includes markers of disease severity on a daily basis. These probabilities are used to calculate an inversed probability weight that is then included as a summary measure of all relevant covariables in the final Cox regression model. However, because marginal structural modeling requires many assumptions that are difficult to be checked, we considered this a sensitivity analysis only.

Data were analyzed with SAS 9.2 (Cary, NC, USA) and R 2.15.1 software (R Foundation for Statistical Computing, Vienna, Austria; packages "etm", "mstate", "ipw").

\section{Results}

We enrolled 544 patients with ARDS who required mechanical ventilation for more than 4 days (Fig. S2). Of these 143 were excluded because of known prior immunocompromise or antiviral treatment and two were excluded because of missing samples. Subsequently, 271 $(68 \%)$ patients tested seropositive for CMV and were thus included in the study. ARDS was of primary pulmonary origin in $158(58 \%)$ of these cases, whereas the remainder was of secondary etiology (e.g., associated with non-pulmonary sepsis, major surgery, or blood transfusion).

\section{CMV reactivation}

CMV reactivation occurred in $74(27 \%)$ of the included patients (Table 1). These patients more frequently hadat the time of ICU admission-concurrent septic shock, higher APACHE IV scores, and renal insufficiency compared to patients who never had CMV reactivation. In addition, a larger proportion of these patients were receiving high dose corticosteroid therapy during the first days in ICU. The median time from ICU admission to onset of reactivation was 8.5 days (interquartile range (IQR) 4-11). Within the subgroup of patients acquiring CMV reactivation the proportion of individuals having relatively high viral loads (at least $1000 \mathrm{IU} / \mathrm{mL}$ ) increased over time (Fig. 1). In a patient population that is selected by an ICU stay of at least 5 weeks, the proportion with CMV viremia is as high as 14 of 23 patients $(61 \%)$.

\section{Clinical outcomes}

On day 30 after study inclusion (this was 35 days following ICU admission) 52 (19\%) patients had died, 209

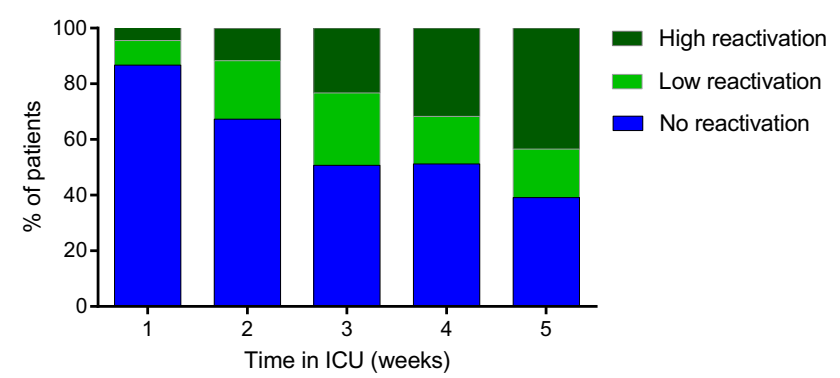

Fig. 1 Viral load in CMV seropositive ARDS patients over time. The quantitative PCR results were calibrated according to the CMV WHO standard; viral loads greater than or equal to $1000 \mathrm{IU} / \mathrm{mL}$ were denoted 'high reactivation'. Viral loads of 100-999 IU/mL were denoted 'low reactivation', and undetectable loads or viral loads below $100 \mathrm{IU} / \mathrm{mL}$ were denoted 'no reactivation' 
Table 2 Crude clinical outcomes of ARDS patients by CMV reactivation status

\begin{tabular}{|c|c|c|c|}
\hline & Reactivation & Non-reactivation & $p$ value \\
\hline Death on ventilator before day $30^{\mathrm{a}}$ & $23 / 74(31)$ & 29/197 (15) & $<0.01$ \\
\hline Death in $\mathrm{ICU}^{\mathrm{b}}$ & $26 / 76(34)$ & $32 / 195(16)$ & $<0.01$ \\
\hline Death by day $90^{\mathrm{b}}$ & $35 / 76(46)$ & $55 / 195(28)$ & $<0.01$ \\
\hline Duration of mechanical ventilation (days) & $15(10-26)$ & $8(6-12)$ & $<0.01$ \\
\hline Length of stay in ICU (days) & $16(11-28)$ & $9(7-14)$ & $<0.01$ \\
\hline
\end{tabular}

a Primary study end point

b CMV reactivation occurred in two additional patients after suc-

(death in ICU, death by day 90). Data show absolute numbers (\%) cessful weaning in the ICU; therefore, 76 instead of 74 patients were considered exposed for analyses with more distal end points

Table 3 Associations between CMV reactivation and clinical outcome

\begin{tabular}{llll}
\hline Analysis & $\begin{array}{l}\text { Successful weaning } \\
\text { (CSHR) }\end{array}$ & $\begin{array}{l}\text { Death on mechanical } \\
\text { ventilation (CSHR) }\end{array}$ & $\begin{array}{l}\text { Death on mechanical } \\
\text { ventilation (SHR) }\end{array}$ \\
\hline $\begin{array}{l}\text { Crude model with adjustment for } \\
\text { time-varying onset of CMV reactivation }\end{array}$ & $0.81(0.58-1.13)$ & $1.75(1.01-3.01)$ & $3.39(1.96-5.87)$ \\
$\begin{array}{l}\text { Multivariable model with adjustment for } \\
\text { time-varying onset of CMV reactivation } \\
\text { baseline imbalances }\end{array}$ & $0.83(0.58-1.18)$ & $1.58(0.86-2.90)$ & $2.74(1.51-4.97)$ \\
$\begin{array}{l}\text { Multivariable model with adjustment for } \\
\text { time-varying onset of CMV reactivation } \\
\text { baseline imbalances }^{\mathrm{a}} \\
\text { evolution of disease prior to onset of CMV reactivation }\end{array}$ & $0.93(0.66-1.31)$ & $1.49(0.78-2.85)$ & $2.48(1.32-4.66)$ \\
\hline
\end{tabular}

Data are presented as hazard ratios with $95 \%$ CI. The causespecific hazard ratio (CSHR) estimates the direct effect of CMV reactivation on clinical outcome (i.e., successful weaning or death on mechanical ventilation). The subdistribution hazard ratio (SHR) is a summary measure of both separate cause-specific hazards and estimates the overall risk of dying from CMV reactivation while taking into account the competing event of successful weaning

a APACHE IV score, use of high dose corticosteroid therapy, and PEEP setting

(77\%) were successfully weaned, and $10(4 \%)$ remained still on mechanical ventilation (Table 2). In crude analyses, patients with CMV reactivation had both a longer duration of mechanical ventilation (15 (IQR 10-26) vs. 8 (IQR 6-12) days; $p<0.01$ ) and higher mortality (23 of $74(31 \%)$ vs. 29 of $197(15 \%)$ patients; $p<0.01)$ compared to subjects without reactivation.

Table 3 shows the results of the various Cox survival regression analyses. Baseline variables associated with reactivation status (at $p<0.30$ ) which changed the crude effect estimate by more than $10 \%$ included the APACHE IV score, use of high dose corticosteroid therapy, and PEEP setting.

In the primary multivariable adjusted analysis, CMV reactivation was no longer statistically associated with either increased mortality or a reduced rate of successful weaning. However, simultaneous effects on both the daily rates of death and weaning did reveal a significant association with overall mortality when competing risks were accounted for (SHR 2.74, $95 \%$ CI 1.51-4.79). As a post hoc sensitivity analysis, we then used marginal structural modeling to assess potential residual b Time-dependent covariables included the risk, injury, failure, loss and end-stage kidney disease (RIFLE) score, Sequential Organ Failure Assessment (SOFA) score, presence of septic shock, and use of high dose corticosteroid therapy, which were all measured on a daily basis until $24 \mathrm{~h}$ prior to reactivation onset

confounding by differences in the evolution of disease severity prior to CMV reactivation between both groups, but found very similar results (Table 3 ). Changing the definitions of our primary end points to include all deaths in the ICU (irrespective of mechanical ventilation status) and discharge (rather than successful weaning) also did not change these findings (Table S1). Furthermore, the independent association with mortality remained among subgroups of patients receiving and not receiving high dose corticosteroid therapy; SHR 2.60 (95\% CI 1.29-5.25) and 3.61 (95\% CI 1.24-10.48), respectively (Table S2). Corticosteroids were mostly used for the treatment of concurrent septic shock (121 of 149 cases).

Figure 2 shows the predicted mortality in a hypothetical population of ARDS patients in which all CMV reactivation is prevented, compared to true (observed) mortality in the study population. The population-attributable fraction of ICU mortality due to CMV reactivation was estimated at $23 \%(95 \%$ CI 6-41\%) by day 30 , which translates into an absolute mortality difference of $4.4 \%$ (95\% CI 1.1-7.9). 


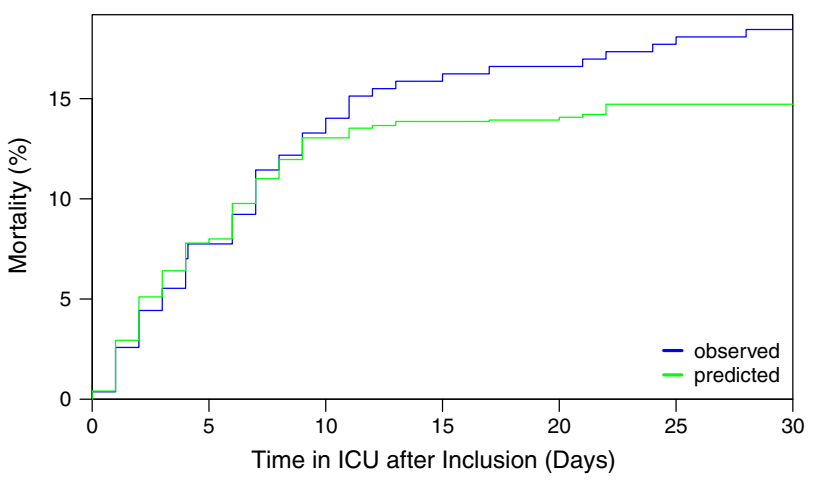

Fig. 2 Observed versus predicted ICU mortality in CMV seropositive ARDS patients. The blue line represents the observed ICU mortality in the study cohort of $271 \mathrm{CMV}$ seropositive patients; the green line represents the predicted ICU mortality if all cases of CMV reactivation in the cohort are prevented. The populationattributable fraction of ICU mortality was $23 \%$ by day 30 (absolute risk difference $4.4 \%$ )

In order to explore possible causal pathways for the observed association between CMV reactivation and death, we performed a post hoc descriptive analysis of the trajectories of organ dysfunction, pulmonary and inflammatory markers over time following reactivation. In short, we compared the 74 patients having CMV reactivation with 74 non-exposed patients who were matched on baseline characteristics and their length of stay in ICU at the onset of reactivation (Table S3). In summary, the total burden of organ dysfunction was slightly higher in patients at the start of CMV reactivation compared to matched non-exposed control subjects, although individual markers of pulmonary dysfunction and inflammation were similar. More importantly, there was a clear trend towards resolution of organ dysfunction over time in nonexposed subjects that was less pronounced in patients having CMV reactivation. However, it should be emphasized that these findings should be interpreted very carefully because of the presence of informative censoring (i.e., patients who die or get discharged do not further contribute to average scores on the group level).

\section{Discussion}

CMV reactivation in ARDS patients increased the overall risk of death on the ventilator through the combined effect of subtle alterations in both the daily rates of death and successful weaning. After accounting for multiple sources of confounding, the absolute mortality that can be attributed to CMV reactivation was estimated to be $4.4 \%$ by day 30 following study inclusion.

Previous findings of excess mortality have triggered debate whether antiviral prophylaxis should be used [29, 30]. However, a greater understanding of pathophysiology and clinical risk factors is necessary to select the optimal target population for such strategies. In our study, reactivation rates were $27 \%$ in ARDS patients overall and $34 \%$ among those with concurrent septic shock. The latter finding might be explained by the increased severity and duration of immune suppression that may be observed in patients with septic shock, including a pronounced depletion of $\mathrm{T}$ cells $[31,32]$. Indeed, a recent study investigating the potential use of antiviral prophylaxis based on the screening of ARDS patients for CMV seroprevalence found that such a strategy is unlikely to be beneficial overall, but suggested a possible benefit in a post hoc subgroup of patients with septic shock [16]. As the proportion of patients with CMV reactivation increased in time, altering the minimal length of stay in the ICU as a criterion may also improve the selection of a high-risk target population. Until then, a pre-emptive treatment strategy (by which patients would be screened for CMV and treated only if reactivation occurs) seems more attractive because the number of patients exposed to the toxicity of (val)ganciclovir would be reduced by $73 \%$. However, the effects of pre-emptive compared to prophylactic treatment on relevant patient outcomes are most likely lower, as treatment is initiated only after reactivation has already begun. Intervention trials comparing prophylaxis, pre-emptive treatment, and wait-andsee strategies are necessary before any evidence-based recommendations regarding the clinical management of CMV reactivation in critically ill patients with ARDS can be made.

Our study has several strengths. First, observations were nested within a large prospective data collection initiative that included consecutive patients, thereby minimizing selection bias [20]. All ARDS events were diagnosed by dedicated trained observers, which minimizes information bias. Moreover, we used a highly sensitive method of quantitative real-time PCR for CMV detection. Most importantly, we used advanced methodologies to account for both competing risks and timedependent information in an attempt to produce unbiased estimates of the independent association between CMV reactivation and clinical outcome.

This methodological approach was mainly necessary because of two reasons. First, Cox regression analysis requires that censoring of survival time must be non-informative, but in our study this was clearly not the case since ARDS patients who are weaned and discharged from the ICU alive are in a better health state than those who remain on the ventilator beyond that time point [33, 34]. Furthermore, when ICU mortality is the event of interest, then discharge must be regarded as a competing event as it precludes this outcome from being observed [14]. The use of the subdistribution hazard model provides a general solution to this informative censoring. Second, the median time to CMV reactivation in our cohort was 8.5 (IQR 4-11) days. If ignored, such delays 
may cause distortion (termed immortal time bias) as nonexposed time observed before the onset of reactivation will be wrongfully attributed to the exposed time at risk, resulting in underestimation of effects associated with CMV reactivation [35, 36]. Time-dependent fitting of CMV reactivation status in our regression models resolved this issue.

Our study also has several limitations. First, even the use of advanced methodology cannot rule out the possibility of unmeasured confounding in an observational study. Therefore, it remains somewhat uncertain whether the excess mortality that we observed can be fully attributed to CMV reactivation, or whether other unknown factors-including other viral reactivations $[8$, 37] - may also be involved. Second, the principle of multivariable analysis to adjust for confounders is to statistically 'force' exposed and non-exposed patients to be similar in all aspects of disease aside from their reactivation status. However, in a dynamic ICU setting, during which critically ill patients continuously deteriorate and improve over time, it is very difficult to verify whether such adjustment was successful. We performed marginal structural modeling as a sensitivity analysis to assess the possible impact of variations in the evolution of disease severity between patients on our effect estimates, yet found very similar results as in our primary analysis. Third, we measured systemic CMV reactivation in plasma but did not collect information about concurrent viral loads in the lungs. This study, therefore, provides no insight into either the prevalence or relevance of pulmonary CMV reactivations. Of note, previous studies have shown that pulmonary reactivation may occur without the concurrent viremia [4, 38, 39]. Furthermore, we focused exclusively on the occurrence of reactivation while patients were on mechanical ventilation (primary analysis) or in the ICU (sensitivity analysis), as we considered these to be the most relevant time windows to potentially treat or prevent CMV reactivation in the ICU. However, because of this deliberate focus we cannot provide information about possible episodes of reactivation that may have occurred later. Likewise we did not investigate the occurrence of reactivations after day 35 in the ICU. Thus, this study only provides insight into the short-term effects of systemic CMV reactivation in ARDS patients in settings in which screening or antiviral prophylaxis is not part of routine clinical practice.
In conclusion, systemic reactivation of CMV in immunocompetent ARDS patients is common and independently associated with death in the ICU. These findings support the need for future studies to better predict CMV reactivation as well as to evaluate the efficacy of treatment strategies directed against CMV reactivation in these patients.

Acknowledgments We thank Huberta Dekker (Department of Medical Microbiology, University Medical Center Utrecht, the Netherlands) for her logistical support in this project, and the participating ICUs and research nurses of the two medical centers for their help in data acquisition. This work was supported by the Center for Translational Molecular Medicine (http://www.ctmm.nl), project MARS (Grant 04I-201). JK received a personal fee from Becton-Dickinson. The sponsor did not play a role in the design and conduct of the study; collection, management, analysis, and interpretation of the data; and preparation, review, or approval of the manuscript; and decision to submit the manuscript for publication.

\section{Compliance with ethical standards}

Conflicts of interest The authors declare that they have no competing interests.

Open Access This article is distributed under the terms of the Creative Commons Attribution-NonCommercial 4.0 International License (http://creativecommons.org/licenses/by-nc/4.0/), which permits any noncommercial use, distribution, and reproduction in any medium, provided you give appropriate credit to the original author(s) and the source, provide a link to the Creative Commons license, and indicate if changes were made.

\section{Appendix}

The MARS Consortium also includes the following persons: Friso M. de Beer, M.D., Lieuwe D. J. Bos, Ph.D., Gerie J. Glas, M.D., Roosmarijn T. M. van Hooijdonk, M.D., Janneke Horn, M.D., Ph.D., Mischa A. Huson, M.D., Laura R. A. Schouten, M.D., Marleen Straat, M.D., Lonneke A. van Vught, M.D., Luuk Wieske, M.D., Ph.D., Maryse A. Wiewel, M.D., Esther Witteveen, M.D. (Academic Medical Center, University of Amsterdam, The Netherlands).

\section{References}

1. Gandhi MK, Khanna R (2004) Human cytomegalovirus: clinical aspects, immune regulation, and emerging treatments. Lancet Infect Dis 4:725-738. doi:10.1016/S1473-3099 (04)01202-2
2. Chiche L, Forel J-M, Roch A et al (2009) Active cytomegalovirus infection is common in mechanically ventilated medical intensive care unit patients. Crit Care Med 37:1850-1857. doi:10.1097/CCM.0b013e31819ffea6
3. Chilet M, Aguilar G, Benet I et al (2010) Virological and immunological features of active cytomegalovirus infection in nonimmunosuppressed patients in a surgical and trauma intensive care unit. J Med Virol 82:1384-1391. doi:10.1002/jmv.21825 
4. Coisel Y, Bousbia S, Forel J-M et al (2012) Cytomegalovirus and herpes simplex virus effect on the prognosis of mechanically ventilated patients suspected to have ventilator-associated pneumonia. PLoS One 7:e51340. doi:10.1371/journal.pone.0051340

5. Heininger A, Haeberle H, Fischer I et al (2011) Cytomegalovirus reactivation and associated outcome of critically ill patients with severe sepsis. Crit Care 15:R77. doi:10.1186/cc10069

6. Kalil AC, Florescu DF (2009) Prevalence and mortality associated with cytomegalovirus infection in nonimmunosuppressed patients in the intensive care unit. Crit Care Med 37:2350-2358. doi:10.1097/CCM. 0b013e3181a3aa43

7. Limaye AP, Kirby KA, Rubenfeld GD et al (2008) Cytomegalovirus reactivation in critically ill immunocompetent patients. JAMA 300:413-422. doi:10.1001/ jama.300.4.413

8. Walton AH, Muenzer JT, Rasche D et al (2014) Reactivation of multiple viruses in patients with sepsis. PLoS One 9:e98819. doi:10.1371/ journal.pone.0098819

9. Ziemann M, Sedemund-Adib B, Reiland P et al (2008) Increased mortality in long-term intensive care patients with active cytomegalovirus infection. Crit Care Med 36:3145-3150. doi:10.1097/CCM.0b013e31818f3fc4

10. Bordes J, Maslin J, Prunet B et al (2011) Cytomegalovirus infection in severe burn patients monitoring by realtime polymerase chain reaction: a prospective study. Burns 37:434-439. doi:10.1016/j.burns.2010.11.006

11. Heininger A, Haeberle H, Fischer I et al (2011) Cytomegalovirus reactivation and associated outcome of critically ill patients with severe sepsis. Crit Care 15:R77. doi:10.1186/cc10069

12. von Müller L, Klemm A, Weiss M et al (2006) Active cytomegalovirus infection in patients with septic shock. Emerg Infect Dis 12:1517-1522. doi: 10.3201/eid1210.060411

13. Jaber S, Chanques G, Borry J et al (2005) Cytomegalovirus infection in critically ill patients: associated factors and consequences. Chest 127:233-241. doi:10.1378/chest.127.1.233

14. Wolkewitz M, Cooper BS, Bonten MJM et al (2014) Interpreting and comparing risks in the presence of competing events. BMJ 349:g5060. doi:10.1136/bmj.g5060
15. Bravo D, Clari MA, Aguilar G et al (2014) Looking for biological factors to predict the risk of active

cytomegalovirus infection in nonimmunosuppressed critically ill patients. J Med Virol 86:827-833. doi:10.1002/jmv.23838

16. Ong DSY, Klein Klouwenberg PMC, Verduyn Lunel FM et al (2015) Cytomegalovirus seroprevalence as a risk factor for poor outcome in acute respiratory distress syndrome. Crit Care Med 43:394-400. doi:10.1097/CCM. 0000000000000712

17. Cook CH, Zhang Y, Sedmak DD et al (2006) Pulmonary cytomegalovirus reactivation causes pathology in immunocompetent mice. Crit Care Med 34:842-849

18. Papazian L, Doddoli C, Chetaille B et al (2007) A contributive result of openlung biopsy improves survival in acute respiratory distress syndrome patients. Crit Care Med 35:755-762. doi:10.1097/01.CCM.0000257325. 88144.30

19. Ong DS, Spitoni C, Klein Klouwenberg P et al (2014) Cytomegalovirus reactivation in critically ill patients with acute respiratory distress syndrome (conference abstract ESICM 2014 Barcelona). Intensive Care Med 40:S127

20. Klein Klouwenberg PMC, Ong DSY, Bos LDJ et al (2013) Interobserver agreement of Centers for Disease Control and Prevention criteria for classifying infections in critically ill patients. Crit Care Med 41:2373-2378. doi:10.1097/CCM.0b013e3182923712

21. Bernard GR, Artigas A, Brigham KL et al (1994) The American-European Consensus Conference on ARDS. Definitions, mechanisms, relevant outcomes, and clinical trial coordination. Am J Respir Crit Care Med 149:818-824

22. van Doornum GJJ, Guldemeester J, Osterhaus ADME, Niesters HGM (2003) Diagnosing herpesvirus infections by real-time amplification and rapid culture. J Clin Microbiol 41:576-580

23. Fine JP, Gray RJ (1999) A proportional hazards model for the subdistribution of a competing risk. J Am Stat Assoc 94:496-509. doi:10.1080/01621459. 1999.10474144

24. Schumacher M, Wangler M, Wolkewitz M, Beyersmann J (2007) Attributable mortality due to nosocomial infections. A simple and useful application of multistate models. Methods Inf Med 46:595-600
25. Beyersmann J, Gastmeier P, Grundmann $\mathrm{H}$ et al (2006) Use of multistate models to assess prolongation of intensive care unit stay due to nosocomial infection. Infect Control Hosp Epidemiol 27:493-499. doi:10.1086/503375

26. Barnett AG, Beyersmann J, Allignol A et al (2011) The time-dependent bias and its effect on extra length of stay due to nosocomial infection. Value Health 14:381-386. doi:10.1016/ j.jval.2010.09.008

27. Robins JM, Hernán MA, Brumback B (2000) Marginal structural models and causal inference in epidemiology. Epidemiology 11:550-560

28. Bekaert M, Timsit J-F, Vansteelandt S et al (2011) Attributable mortality of ventilator-associated pneumonia: a reappraisal using causal analysis. Am J Respir Crit Care Med 184:1133-1139. doi:10.1164/rccm.201105-0867OC

29. Forel J-M, Martin-Loeches I, Luyt C-E (2014) Treating HSV and CMV reactivations in critically ill patients who are not immunocompromised: pro. Intensive Care Med 40:1945-1949. doi: 10.1007/s00134-014-3445-y

30. Chanques G, Jaber S (2014) Treating HSV and CMV reactivations in critically ill patients who are not immunocompromised: con. Intensive Care Med 40:1950-1953. doi: 10.1007/s00134-014-3521-3

31. Hotchkiss RS, Monneret G, Payen D (2013) Sepsis-induced immunosuppression: from cellular dysfunctions to immunotherapy. Nat Rev Immunol 13:862-874. doi: $10.1038 /$ nri3552

32. Campbell J, Trgovcich J, Kincaid M et al (2012) Transient CD8-memory contraction: a potential contributor to latent cytomegalovirus reactivation. J Leukoc Biol 92:933-937. doi: 10.1189/jlb.1211635

33. Bekaert M, Vansteelandt S, Mertens K (2010) Adjusting for time-varying confounding in the subdistribution analysis of a competing risk. Lifetime Data Anal 16:45-70. doi: 10.1007/s10985-009-9130-8

34. Wolkewitz M, Beyersmann J, Gastmeier P, Schumacher M (2009) Modeling the effect of time-dependent exposure on intensive care unit mortality. Intensive Care Med 35:826-832. doi:10.1007/s00134009-1423-6

35. Shintani AK, Girard TD, Eden SK et al (2009) Immortal time bias in critical care research: application of timevarying Cox regression for observational cohort studies. Crit Care Med 37:2939-2945. doi:10.1097/ CCM.0b013e3181b7fbbb 
36. Suissa S (2003) Effectiveness of inhaled corticosteroids in chronic obstructive pulmonary disease: immortal time bias in observational studies. Am J Respir Crit Care Med 168:49-53. doi: $10.1164 / \mathrm{rccm}$. 200210-1231OC

37. Roa PL, Hill JA, Kirby KA et al (2015) Coreactivation of human herpesvirus 6 and cytomegalovirus is associated with worse clinical outcome in critically ill adults. Crit Care Med. doi:

10.1097/CCM.0000000000000969
38. Blanquer J, Chilet $\mathrm{M}$, Benet I et al (2011) Immunological insights into the pathogenesis of active CMV infection in non-immunosuppressed critically ill patients. J Med Virol 83:1966-1971. doi:10.1002/jmv.22202
39. Friedrichs I, Bingold T, Keppler OT et al (2013) Detection of herpesvirus EBV DNA in the lower respiratory tract of ICU patients: a marker of infection of the lower respiratory tract? Med Microbiol Immunol 202:431-436. doi: 10.1007/s00430-013-0306-1 